\title{
Comparison Based Diagnostics as a Probabilistic Deduction Problem
}

\author{
Balázs Polgár \\ Dept. of Measurement and Inf. Systems, BUTE \\ Magyar Tudósok krt. 2, Budapest, Hungary, H-1117, \\ polgar@mit.bme.hu
}

\begin{abstract}
A novel modeling approach for system-level diagnosis of multiprocessor systems has been introduced in previous publications. In this approach the diagnostic process is formulated as an optimization problem. The possible logical relations are identified between the different pieces of diagnostic information of the system and an optimal consistent combination of the relations is determined during the solution method. A part of the information is that, which can be observed at the outputs of the system. Another part is composed by hypotheses on the states of units. Relations between these information are described by consequence rules having probabilities assigned to them. These probabilities express the uncertainty of test results. The object is to draw back the set of observed information to a subset of hypotheses on unit states with the maximum likelihood, i.e. to determine the states of system units on the basis of the syndrome.
\end{abstract}

\section{Introduction}

Diagnostics is one of the major tools for assuring the reliability of complex systems in information technology. In such systems the test process is often implemented on system-level: main components are tested, results are collected, and based on this information the good or faulty state of each system-component is determined. This classification procedure is known as diagnostic process.

The early approaches employed oversimplified models, but these proved to be impractical, lately much effort has been put into extending the limitations of traditional models [1. However, the presented solutions mostly concentrated on only one aspect of the problem.

Application of P-graph based modeling to system-level diagnosis 2] can provide a general framework that supports the solution of several different types of problems, that previously needed numerous different modeling approaches and solution algorithms. Furthermore, we have not only integrated existing solution methods, but proceeding from a more general base we have extended the set of solvable problems with new ones. The representational power of the model was illustrated in paper [3].

Another advantage of the $\mathrm{P}$-graph models is that it takes into consideration more properties of the real system than previous diagnostic models. Therefore 
its diagnostic accuracy is also better. This means that it provides almost good diagnosis even when half of the processors are faulty [3], which is important for example in the field of wafer scale testing 4]. These favorable properties of the approach are achieved by considering the diagnostic system as a structured set of information called hypotheses with well-defined relations.

In part of the paper the adapted abstract model is presented in order to be able to reuse or apply it to other fields of diagnostics. The application for comparison based diagnostics and an example is also discussed.

\section{Comparison Based System-Level Diagnostics in Multiprocessor Systems}

System-level diagnosis considers the replaceable units of a system, and does not deal with the exact location of faults within these units. A system consists of an interconnected network of independent but cooperating units (typically processors, the elements of set $\mathbf{U})$. These units can fail, therefore a diagnostic procedure is performed from time to time to ensure the correct behavior of the whole system. According to this the fault state of each unit is considered as either good or faulty (the domain of the states $\mathbf{D}_{\text {st }}=\{g, f\}$ and the function $s t(u)$ determines the actual state of unit $u \in \mathbf{U})$. The collection of the fault states of every unit in the system is called the fault pattern [6].

A possible way to detect the failures of the units in multiprocessor systems is the comparison based diagnostics [7]. This is used for instance in the commercially available APEMille supercomputer [8] which was developed in collaboration by IEI-CNR of Rome and Pisa, and the DESY Zeuthen in Germany. A further possible application field of this model is the wafer scale diagnosis [9].

In the comparison based approach the system consists comparators between pairs of the homogeneous units. Both units perform the same test and the comparator compares the bit-sequence result. The set of comparators is denoted by $\mathbf{C}$ and the set of tests is denoted by $\mathbf{T} \subset \mathbf{U} \times \mathbf{C} \times \mathbf{U}(\times$ denotes the Cartesian product of two sets), which has the elements $(A, c, B)$, where units $A$ and $B$ are neighbors and have comparator $c$ between them for comparing their testsequence results. The so-called syndrome contains the results of the comparators, namely the information that 'the two units differ' or 'the two units operate similarly' denoted by 1 and 0 , respectively. Formally, $\mathbf{D}_{\mathbf{t r}}=\{0,1\}$ is the domain of the test results and function $\operatorname{tr}(t)$ determines the actual test result of test $t \in \mathbf{T}$. The object is to determine the fault pattern on the basis of the syndrome.

If comparators are assumed to be fault free then the test result is always 0 if both unit is good, it is always 1 if one is good and the other is faulty and it can be either 0 or 1 if both is faulty. The behavior in the last case can be described by assigning probabilities to test results, see Table 10 [3].

The diagnostics is more complicated if possible faults of comparators should also be taken into consideration. The behavior of a faulty comparator can be described by the parameters $p_{C 00}, p_{C 01}, p_{C 10}, p_{C 11}$, where $p_{C x y}$ is the probability that a faulty comparator alters the expected test result from $x$ to $y$ (Table 2). 
Table 1. Probabilities of test results depending on unit states if fault free comparators are assumed

\begin{tabular}{|c|c|c|c|}
\hline \multirow{2}{*}{$\begin{array}{c}\text { State } \\
\text { of } A\end{array}$} & \multirow{2}{*}{$\begin{array}{c}\text { State } \\
\text { of } B\end{array}$} & \multicolumn{2}{|c|}{ Prob. of testres } \\
\hline & & 0 & 1 \\
\hline good & good & 1 & 0 \\
\hline good & faulty & 0 & 1 \\
\hline faulty & good & 0 & 1 \\
\hline faulty & faulty & $p_{d 0}$ & $p_{d 1}$ \\
\hline
\end{tabular}

Table 2. Behavior of a comparator that can be faulty

\begin{tabular}{|c||c|c|c|c|}
\hline \multicolumn{1}{|c|}{$\begin{array}{c}\text { State } \\
\text { of the } \\
\text { comp. }\end{array}$} & \multicolumn{4}{c|}{$\begin{array}{c}\text { Probability of altering } \\
\text { test result from } \mathbf{x} \text { to } \mathbf{y}\end{array}$} \\
\cline { 2 - 5 } good & $0 \rightarrow 0$ & $0 \rightarrow 1$ & $1 \rightarrow 0$ & $1 \rightarrow 1$ \\
faulty & 1 & 0 & 0 & 1 \\
$p_{C 00}$ & $p_{C 01}$ & $p_{C 10}$ & $p_{C 11}$ \\
\hline
\end{tabular}

\section{Basic Terminology and Its Application}

In this section basic definitions and notations are presented. A part of it (for instance the concept of $\mathrm{P}$-graphs, decision mappings and their relation) is taken directly from the field of PNS problems, introduced by Friedler et al. in [10]. Another part is taken from there with an adapted terminology and with some modified interpretation (for instance hypotheses instead of materials, logical relations instead of operating units). The model consists some additional properties, too (e.g. excluding hypotheses).

\subsection{Hypotheses and Their Relations}

Hypotheses are said to be a kind of statements or assumptions, that contain some information about the system, but the truth of which aren't necessarily known. Hypotheses can be for instance about the possible states of units or about the possible outcomes of tests. The probability that hypothesis $h$ becomes true is determined by the function $p(h)$.

Considering the set $\mathbf{H}$ of hypotheses, logical relations between hypotheses can be defined as follows. The pair of $\left(\mathbf{h}_{\mathbf{p}}, \mathbf{h}_{\mathbf{c}}\right), \mathbf{h}_{\mathbf{p}} \subset \mathbf{H}, \mathbf{h}_{\mathbf{c}} \subset \mathbf{H}$ describes a probabilistic consequence relation, where $\mathbf{h}_{\mathbf{p}}$ is the set of premisses, $\mathbf{h}_{\mathbf{c}}$ is the set of consequences and the probability function $p\left(\left(\mathbf{h}_{\mathbf{p}}, \mathbf{h}_{\mathbf{c}}\right)\right)$ determines the conditional probability that hypotheses in set $\mathbf{h}_{\mathbf{c}}$ become true if hypotheses in set $\mathbf{h}_{\mathbf{p}}$ are supposed to be true.

Function exc $: \mathbf{H} \rightarrow 2^{\mathbf{H}}$ determines the excluded sets of hypotheses for the elements of $\mathbf{H}$, that is for hypothesis $h$ the set $\boldsymbol{e x}(h)$ contains those hypotheses, which cannot be true if $h$ is true (function $2^{\mathbf{H}}$ determines the power set of set $\mathbf{H}$, i.e. the set of all subsets of the given set). With this function for instance that kind of constraints can be formalized, which assure the unique state of a unit.

Application in Comparison Based Diagnostics. Four sets of hypotheses can be distinguished in the diagnostic model described in section 2 ;

- hypotheses about the states of units

$$
\begin{array}{r}
\mathbf{H}_{\mathbf{1}}=\left\{A_{g} \mid A_{g} \equiv{ }^{\prime} \operatorname{st}(A)=g^{\prime} \equiv \text { 'unit } A \text { is good', } A \in \mathbf{U}\right\} \\
\cup\left\{A_{f} \mid A_{f} \equiv{ }^{\prime} \operatorname{st}(A)=f ' \equiv \text { 'unit } A \text { is faulty', } A \in \mathbf{U}\right\}
\end{array}
$$


- hypotheses about the states of comparators

$$
\mathbf{H}_{\mathbf{2}}=\left\{c_{g} \mid c_{g} \equiv{ }^{\prime} s t(c)=g^{\prime}, c \in \mathbf{C}\right\} \cup\left\{c_{f} \mid c_{f} \equiv{ }^{\prime} s t(c)=f^{\prime}, c \in \mathbf{C}\right\}
$$

- hypotheses about the comparison results if comparator faults are not taken into consideration

$$
\begin{aligned}
\mathbf{H}_{\mathbf{3}}= & \left\{A B_{0} \mid A B_{0} \equiv ' \operatorname{tr}((A, c, B))=0 \& \operatorname{st}(c)=g ' \equiv \text { 'unit } A\right. \\
& \text { and unit } B \text { are in the same state', } A, B \in \mathbf{U}, c \in \mathbf{C} \text { and }(A, c, B) \in \mathbf{T}\} \\
& \cup\left\{A B_{1} \mid A B_{1} \equiv ' \operatorname{tr}((A, c, B))=1 \& \operatorname{st}(c)=g ' \equiv \text { 'unit } A\right. \\
& \text { and unit } B \text { are in different states', } A, B \in \mathbf{U}, c \in \mathbf{C} \text { and }(A, c, B) \in \mathbf{T}\}
\end{aligned}
$$

- hypotheses about the comparison results if comparator faults can alter the real results

$$
\begin{array}{r}
\mathbf{H}_{\mathbf{4}}=\left\{A c B_{0} \mid A c B_{0} \equiv{ }^{\prime} \operatorname{tr}((A, c, B))=0^{\prime}, A, B \in \mathbf{U}, c \in \mathbf{C}, \text { and }(A, c, B) \in \mathbf{T}\right\} \\
\cup\left\{A c B_{1} \mid A c B_{1} \equiv{ }^{\prime} \operatorname{tr}((A, c, B))=1^{\prime}, A, B \in \mathbf{U}, c \in \mathbf{C}, \text { and }(A, c, B) \in \mathbf{T}\right\}
\end{array}
$$

Consequence relations formalize the information given in Tables 1 and 2

$$
\mathbf{R}_{\mathbf{1}}=\left\{\left(\left\{A_{s}, B_{t}\right\},\left\{A B_{x}\right\}\right) \mid A_{s}, B_{t} \in \mathbf{H}_{\mathbf{1}}, A B_{x} \in \mathbf{H}_{\mathbf{3}}\right\}
$$

An element $r$ of this set having probability $p(r)$ represents the relation 'if $A$ is in state $s, B$ is in state $t$ and there exists a fault free comparator between them then the test result is $x$ with probability $p(r)^{\prime}$. The probability function $p(\cdot)$ for these relations is determined by the parameters given in Table 1 .

$$
\mathbf{R}_{\mathbf{2}}=\left\{\left(\left\{A B_{x}, c_{s}\right\},\left\{A c B_{y}\right\}\right) \mid c_{s} \in \mathbf{H}_{\mathbf{2}}, A B_{x} \in \mathbf{H}_{\mathbf{3}}, A c B_{y} \in \mathbf{H}_{\mathbf{4}}\right\}
$$

An element $r$ of this set having probability $p(r)$ represents the relation 'if the expected test result is $x$ and comparator $c$ is in state $s$ then the real test result is $y$ with probability $p(r)^{\prime}$ '. The probability function $p(\cdot)$ for these relations is determined by the parameters given in Table 2 .

The excluded set of hypotheses for a hypothesis $h \in \mathbf{H}_{\mathbf{1}} \cup \mathbf{H}_{\mathbf{2}}$ consists all hypotheses representing another assumption on the state of the given unit or comparator: $\boldsymbol{e} \boldsymbol{x c}\left(A_{s}\right)=\left\{A_{t} \mid t \in \mathbf{D}_{\text {st }} \backslash\{s\}\right\}$.

Example. Let us consider a system with three units $(A, B, C)$ and three comparators $\left(c^{1}, c^{2}, c^{3}\right)$ according to Fig. 1 where both units and comparators can fail. The set of hypotheses is $\mathbf{H}=\mathbf{H}_{\mathbf{1}} \cup \mathbf{H}_{\mathbf{2}} \cup \mathbf{H}_{\mathbf{3}} \cup \mathbf{H}_{\mathbf{4}}$, where

$$
\begin{aligned}
& \mathbf{H}_{\mathbf{1}}=\left\{A_{g}, A_{f}, B_{g}, B_{f}, C_{g}, C_{f}\right\} \\
& \mathbf{H}_{\mathbf{2}}=\left\{c_{g}^{1}, c_{f}^{1}, c_{g}^{2}, c_{f}^{2}, c_{g}^{3}, c_{f}^{3}\right\} \\
& \mathbf{H}_{\mathbf{3}}=\left\{A B_{0}, A B_{1}, B C_{0}, B C_{1}, A C_{0}, A C_{1}\right\} \\
& \mathbf{H}_{\mathbf{4}}=\left\{A c^{1} B_{0}, A c^{1} B_{1}, B c^{2} C_{0}, B c^{2} C_{1}, A c^{3} C_{0}, A c^{3} C_{1}\right\}
\end{aligned}
$$




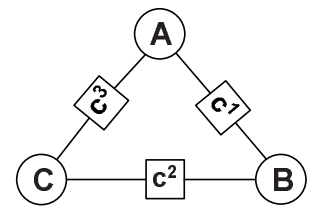

Fig. 1. System with units $A, B, C$ and comparators $c^{1}, c^{2}, c^{3}$

The set of consequence relations is $\mathbf{R}=\mathbf{R}_{\mathbf{1}} \cup \mathbf{R}_{\mathbf{2}}$, where

$$
\begin{aligned}
\mathbf{R}_{1}= & \left\{\left(\left\{A_{g}, B_{g}\right\},\left\{A B_{0}\right\}\right),\left(\left\{A_{g}, B_{f}\right\},\left\{A B_{1}\right\}\right),\left(\left\{A_{f}, B_{g}\right\},\left\{A B_{1}\right\}\right),\right. \\
& \left.\left(\left\{A_{f}, B_{f}\right\},\left\{A B_{0}\right\}\right),\left(\left\{A_{f}, B_{f}\right\},\left\{A B_{1}\right\}\right), \ldots\right\} \\
\mathbf{R}_{2}= & \left\{\left(\left\{A B_{0}, c_{g}^{1}\right\},\left\{A c^{1} B_{0}\right\}\right),\left(\left\{A B_{0}, c_{f}^{1}\right\},\left\{A c^{1} B_{0}\right\}\right),\left(\left\{A B_{0}, c_{f}^{1}\right\},\left\{A c^{1} B_{1}\right\}\right), \ldots\right\}
\end{aligned}
$$

The excluded sets of hypotheses - where it is not empty- are according to the followings: $\boldsymbol{e x c}\left(A_{g}\right)=\left\{A_{f}\right\}, \boldsymbol{e x c}\left(A_{f}\right)=\left\{A_{g}\right\}, \boldsymbol{e x c}\left(B_{g}\right)=\left\{B_{f}\right\} \ldots$

\subsection{Probabilistic Deduction Problem}

The probabilistic deduction problem ( $\mathrm{PD}$ problem, for short) based on set $\mathbf{H}$ of hypotheses is defined by the triplet $\left(\mathbf{H}^{\mathbf{i}}, \mathbf{H}^{\mathbf{d}}, \mathbf{R}\right)$, where $\mathbf{H}^{\mathbf{i}} \subset \mathbf{H}$ is the set of initial hypotheses, $\mathbf{H}^{\mathbf{d}} \subset \mathbf{H}$ is the set of hypotheses to be deduced and $\mathbf{R} \subseteq 2^{\mathbf{H}} \times 2^{\mathbf{H}}$ is the set of probabilistic consequence relations between hypotheses. The object is to deduce the elements of set $\mathbf{H}^{\mathbf{d}}$ from a subset of $\mathbf{H}^{\mathbf{i}}$.

In the probabilistic deduction problem $\left(\mathbf{H}^{\mathbf{i}}, \mathbf{H}^{\mathbf{d}}, \mathbf{R}\right)$ the base set of hypotheses $\mathbf{H}^{\mathbf{b}}$ is the set of all hypotheses defined implicitly by the triplet $\left(\mathbf{H}^{\mathbf{i}}, \mathbf{H}^{\mathbf{d}}, \mathbf{R}\right)$, i.e.

$$
\mathbf{H}^{\mathbf{b}}=\mathbf{H}^{\mathbf{i}} \cup \mathbf{H}^{\mathbf{d}} \cup\left\{h \mid h \in \mathbf{h}_{\mathbf{p}} \cup \mathbf{h}_{\mathbf{c}}, \quad\left(\mathbf{h}_{\mathbf{p}}, \mathbf{h}_{\mathbf{c}}\right) \in \mathbf{R}\right\}
$$

Application in Comparison Based Diagnostics. First the model consisting fault-free comparators is considered. Let set $\mathbf{H}_{1}^{\mathbf{d}}$ be defined as that subset of $\mathbf{H}_{\mathbf{3}}$ which corresponds to the syndrome determined by function $\operatorname{tr}: \mathbf{H}_{1}^{\mathbf{d}}=\left\{A B_{x} \mid\right.$ $x=\operatorname{tr}((A, c, B))$ and $\left.A B_{x} \in \mathbf{H}_{\mathbf{3}}\right\}$. Then the diagnostic task is formulated as the PD problem $\left(\mathbf{H}_{\mathbf{1}}, \mathbf{H}_{\mathbf{1}}^{\mathbf{d}}, \mathbf{R}_{\mathbf{1}}\right)$.

If comparator faults are also included in the model, then the diagnostic task is formulated as PD problem $\left(\mathbf{H}_{\mathbf{1}} \cup \mathbf{H}_{\mathbf{2}}, \mathbf{H}_{\mathbf{2}}^{\mathrm{d}}, \mathbf{R}_{\mathbf{1}} \cup \mathbf{R}_{\mathbf{2}}\right)$, where $\mathbf{H}_{\mathbf{2}}^{\mathrm{d}}=\left\{A c B_{x} \mid\right.$ $x=\operatorname{tr}((A, c, B))$ and $\left.A c B_{x} \in \mathbf{H}_{\mathbf{4}}\right\}$

The base set of hypotheses for the first model is $\mathbf{H}_{\mathbf{1}}^{\mathbf{b}}=\mathbf{H}_{\mathbf{1}} \cup \mathbf{H}_{\mathbf{3}}$, whereas for the second one it is $\mathbf{H}_{2}^{\mathrm{b}}=\mathbf{H}_{\mathbf{1}} \cup \mathbf{H}_{2} \cup \mathbf{H}_{3} \cup \mathbf{H}_{4}$.

Example (continued). Let's consider that the syndrome is 101, i.e. $\operatorname{tr}\left(\left(A, c^{1}, B\right)\right)=1, \operatorname{tr}\left(\left(B, c^{2}, C\right)\right)=0$ and $\operatorname{tr}\left(\left(A, c^{3}, C\right)\right)=1$. The set of hypotheses to be deduced for the example introduced above is $\mathbf{H}_{2}^{\mathbf{d}}=$ $\left\{A c^{1} B_{1}, B c^{2} C_{0}, A c^{3} C_{1}\right\}$. 


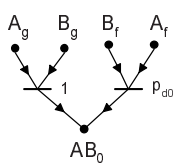

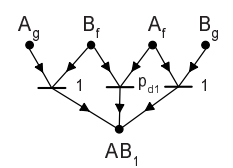

(a)
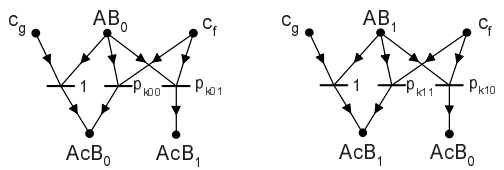

(b)

Fig. 2. P-graph model of a single comparator test

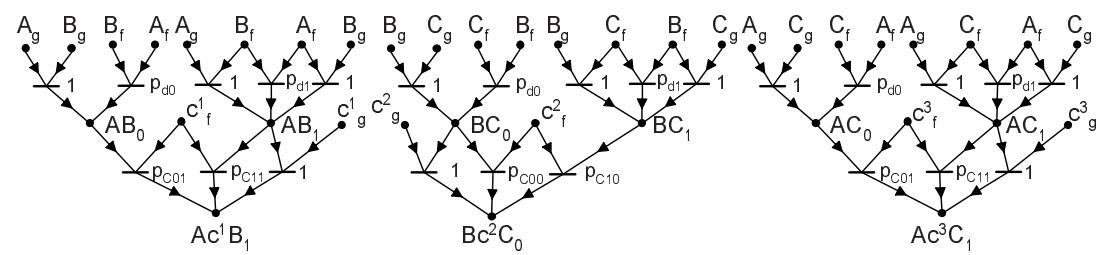

Fig. 3. P-graph model of the example (corresponding to the syndrome)

\subsection{P-Graphs}

For graphical representation of the probabilistic deduction problem the so-called $\mathrm{P}$-graphs are used.Consider two finite sets $\mathbf{h}$ and $\mathbf{r}$ such that $\mathbf{r} \subseteq 2^{\mathbf{h}} \times 2^{\mathbf{h}}$. A $P$-graph is defined as a directed bipartite graph that is determined by the pair of $(\mathbf{h}, \mathbf{r})$ by the following way:

1. the vertices of the graph are the elements of the set $\mathbf{V}=\mathbf{h} \cup \mathbf{r}$; vertices belonging to set $\mathbf{h}$ are called H-type and are denoted by a dot, whereas those belonging to set $\mathbf{r}$ are called $R$-type and are denoted by a horizontal bar;

2. the arcs of the graph are the elements of set $\mathbf{E}=\mathbf{E}_{\mathbf{1}} \cup \mathbf{E}_{\mathbf{2}}$, where $\mathbf{E}_{\mathbf{1}}$ and $\mathbf{E}_{\mathbf{2}}$ are the sets of arcs from the premisses to the relations and from the relations to the consequences, respectively. I.e., $\mathbf{E}_{\mathbf{1}}=\left\{(h, r) \mid r=\left(\mathbf{h}_{\mathbf{1}}, \mathbf{h}_{\mathbf{2}}\right) \in\right.$ $\mathbf{r}$ and $\left.h \in \mathbf{h}_{\mathbf{1}}\right\}$ and $\mathbf{E}_{\mathbf{2}}=\left\{(r, h) \mid r=\left(\mathbf{h}_{\mathbf{1}}, \mathbf{h}_{\mathbf{2}}\right) \in \mathbf{r}\right.$ and $\left.h \in \mathbf{h}_{\mathbf{2}}\right\}$.

Application in Comparison Based Diagnostics. The P-graph of a single comparison test between units $A$ and $B$ with comparator $c$ can be seen on Fig. 2(a) if comparators are assumed to be fault-free. This P-graph should be extended with the graph on Fig. 2(b) if comparator faults are taken into consideration. The labels beside the H-type vertices are the names of the hypotheses, whereas beside the R-type vertices the probability of the relation can be seen.

Example (cont.). Part of the P-graph model of the hypotheses and relations defined previously, which corresponds to the syndrome can be seen on Fig. 3 . 


\subsection{Deduction Structures}

Consider the probabilistic deduction problem $\left(\mathbf{H}^{\mathbf{i}}, \mathbf{H}^{\mathbf{d}}, \mathbf{R}\right)$ and its base set $\mathbf{H}^{\mathbf{b}}$. Let $\mathbf{h} \subseteq \mathbf{H}^{\mathbf{b}}$ and $\mathbf{r} \subseteq \mathbf{R}$ be two sets such that $\mathbf{r} \subseteq 2^{\mathbf{h}} \times 2^{\mathbf{h}}$. Then the structure consisting the set of hypotheses $\mathbf{h}$ and the set of relations $\mathbf{r}$ between them is called a deduction structure of $\left(\mathbf{H}^{\mathbf{i}}, \mathbf{H}^{\mathbf{d}}, \mathbf{R}\right)$ and is formally determined by the $\mathrm{P}$ graph $(\mathbf{h}, \mathbf{r})$. A deduction structure given by a $\mathrm{P}$-graph $(\mathbf{h}, \mathbf{r})$ is called consistent if it does not consist hypotheses that exclude each other, i.e. $\mathbf{h} \cap \boldsymbol{e x c}[\mathbf{h}]=\emptyset$.

Example (cont.). The P-graph on Fig. 3 and all of its subgraphs are deduction structures. The P-graphs on Fig. 4 are consistent deduction structures.

\subsection{Solution Structures}

The deduction structure given by $\mathrm{P}$-graph $(\mathbf{h}, \mathbf{r})$ is called a solution structure of the probabilistic deduction problem $\left(\mathbf{H}^{\mathbf{i}}, \mathbf{H}^{\mathbf{d}}, \mathbf{R}\right)$ if

1. it is consistent,

2. it consist all hypotheses to be deduced, i.e. $\mathbf{H}^{\mathbf{d}} \subset \mathbf{h}$.

3. a vertex $h$ of H-type has no arc pointing to it if and only if it corresponds to a hypothesis belonging to the set of initial hypotheses.

4. a vertex $h$ of H-type has no arc starting from it if and only if it corresponds to a hypothesis belonging to the set of hypotheses to be deduced.

5. every vertex of R-type has at least one path leading to a vertex of H-type representing a hypotheses to be deduced.

Let $\mathbf{S}\left(\mathbf{H}^{\mathbf{i}}, \mathbf{H}^{\mathbf{d}}, \mathbf{R}\right)$ denote the set of all solution structure of the probabilistic deduction problem $\left(\mathbf{H}^{\mathbf{i}}, \mathbf{H}^{\mathbf{d}}, \mathbf{R}\right)$. The weight function $W(s)$ assigns a value to every solution structure $s \in \mathbf{S}\left(\mathbf{H}^{\mathbf{i}}, \mathbf{H}^{\mathbf{d}}, \mathbf{R}\right)$. This function serves as the optimization objective function during the selection between solution structures.

Application in Comparison Based Diagnostics. The initial sets of hypotheses in solution structures represent the fault patterns that is compatible with the syndrome. The object is to select one of it, which is optimal according to a given criteria. This criteria is determined by the weight function, which can be for instance the conditional probabilities of the syndrome given the conditions of the possible fault patterns. In PD problem for a solution structure $\left(\mathbf{h}_{\mathbf{s}}, \mathbf{r}_{\mathbf{s}}\right) \in \mathbf{S}\left(\mathbf{H}^{\mathbf{i}}, \mathbf{H}^{\mathbf{d}}, \mathbf{R}\right)$, this is the probability of $\mathbf{P}\left(\mathbf{H}^{\mathbf{d}} \mid \mathbf{H}^{\mathbf{i}} \cap \mathbf{h}_{\mathbf{s}}\right)=\mathbf{P}$ (syndrome $\mid$ fault pattern). In this case we are talking about maximum likelihood diagnostics, because that fault pattern is chosen by which the occurrence of the given syndrome has the maximal probability.

In comparison based diagnostics if fault-free comparators are assumed the former version of the weight function is determined according to the formula:

$$
\begin{aligned}
W\left(\left(\mathbf{h}_{\mathbf{s}}, \mathbf{r}_{\mathbf{s}}\right)\right) & =\mathbf{P}\left(\mathbf{H}^{\mathbf{d}} \mid \mathbf{H}^{\mathbf{i}} \cap \mathbf{h}_{\mathbf{s}}\right)=\bigcap_{h \in \mathbf{H}^{\mathbf{d}}} \mathbf{P}\left(h \mid \mathbf{H}^{\mathbf{i}} \cap \mathbf{h}_{\mathbf{s}}\right)= \\
& =\bigcap_{h \in \mathbf{H}^{\mathbf{d}} ;\left(\mathbf{h}_{\mathbf{p}},\{h\}\right) \in \mathbf{r}_{\mathbf{s}}} \mathbf{P}\left(h \mid \mathbf{h}_{\mathbf{p}}\right)=\bigcap_{r \in \mathbf{r}_{\mathbf{s}}} p(r)
\end{aligned}
$$


By this definition, the solution structure having the maximum value of the weight function should be determined. It can be transformed into a minimization problem if necessary by taking the sums of the negative logarithms of the probabilities of relations as weight function.

Example. Two feasible solution structures $s_{1}$ and $s_{2}$ can be seen on Fig. 4(a) and Fig. 4(b). The weights of these structures are the products of the probabilities of the relations included. For $s_{1}$ it is 1 , meaning that for the given fault pattern it is sure that the considered syndrome will be the result of the tests. The weight of $s_{2}$ is $p_{C 01} \cdot p_{C 01}$ meaning that for this fault pattern the syndrome arise with this probability. In maximum likelihood diagnostics that fault pattern is 'selected' for which the syndrome arise with the maximum likelihood. This means that if probability parameters in Tables 1 and 2 are smaller than 1 than the diagnosis will be the fault pattern according to $s_{1}$.

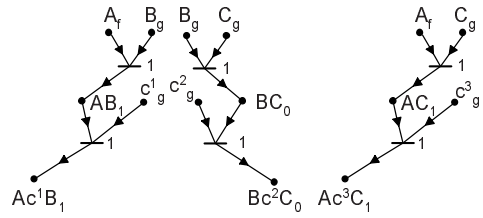

(a)

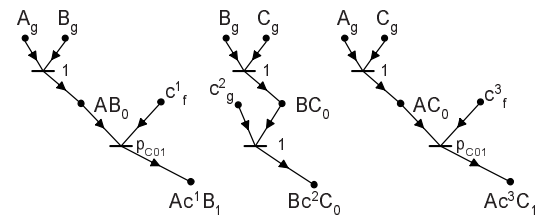

(b)

Fig. 4. Two feasible solution structures of the example

\section{Conclusions}

In this paper a formal description of the previously introduced model of P-graph based diagnostics is presented. In probabilistic deduction problems the relations of the information is modeled, which relations corresponds to the structure of the system and to the structure of the diagnostic procedure. Solving this model the most probable states of units can be determined on the basis of the syndrome. The concept of the model -formulating in general- is that knowing the relations between pieces of information and knowing the observed information it is possible to deduce for the hidden or embedded information with the maximum likelihood (an information is considered hidden or embedded if it cannot be observed directly). The PD problem can be solved either with a general solver for linear programming problems or with the adapted version of the solution algorithm of PNS problems developed by Friedler et al. 10. The model and its solution algorithm have been already implemented in $\mathrm{C}++$, and simulation results demonstrate its good diagnostic performance [3]. 


\section{References}

1. S. N. Maheshwari, S. L. Hakimi. On Models for Diagnosable Systems and Probabilistic Fault Diagnosis. IEEE Trans. on Computers, vol. C-25, pp. 228-236, 1976.

2. B. Polgár, Sz. Nováki, A. Pataricza, F. Friedler. A Process-Graph Based Formulation of the Syndrome-Decoding Problem, In 4 th Workshop on Design and Diagnostics of Electronic Circuits and Systems, pp. 267-272, Hungary, 2001.

3. B. Polgár, T. Bartha, E. Selényi. On the Extension and Applicability of the P-Graph Modeling Paradigm to System-Level Diagnostic Problems, invited and submitted to PDCP Special Issue of DAPSYS'2002 Workshop.

4. S. Chessa. Self-Diagnosis of Grid-Interconnected Systems, with Application to Self-Test of VLSI Wafers, Ph.D. Thesis, TD-2/99 Univ. of Pisa, Italy, March 1999.

5. F. Friedler, L. T. Fan, And B. Imreh. Process Network Synthesis: Problem Definition. Networks, 28(2), pp. 119-124, 1998.

6. M. Barborak, M. Malek, and A. Dahbura. The Consensus Problem in Fault Tolerant Computing, ACM Computing Surveys, vol. 25, pp. 171-220, June 1993.

7. A. Dahbura, K. Sabnani, and L. King. The Comparison Approach to Multiprocessor Fault Diagnosis, IEEE Trans. on Computers, vol. C-36, pp. 373-378, 1987.

8. F. Aglietti, et AL.. Self-Diagnosis of APEmille, Proc. EDCC-2 Companion Workshop on Dependable Computing, pp. 73-84, Silesian Technical University, Gliwice Poland, May 1996.

9. B. Sallay, P. Maestrini, P. Santi. Comparison-Based Wafer-Scale Diagnosis Tolerating Comparator Faults, IEEE Journal on Computers and Digital Techniques, 146(4), pp. 211-215, 1999.

10. F. Friedler, K. Tarjan, Y. W. Huang, L. T. Fan. Combinatorial Algorithms for Process Synthesis. Comp. in Chemical Engineering, vol. 16, pp. 313-320, 1992. 